\title{
Shape and Volume of anti-Poly-D-alanyl Antibodies in the Presence and Absence of Tetra-D-alanine as Followed by Small-Angle X-ray Scattering
}

\author{
By I. PILz \\ Institut für Physikalische Chemie der Universität, Heinrichstrasse 28, 8010 Graz, Austria \\ O. KRATKY \\ Institut für Röntgenfeinstrukturforschung, Steyrergasse 17, 8010-Graz, Austria \\ AND A. Licht AND M. Sela \\ The Weizmann Institute of Science, Department of Chemical Immunology, Rehovot, Israel
}

\begin{abstract}
The conformation of two anti-poly-D-alanyl antibodies was studied before and after interaction with hapten by small-angle $\mathrm{X}$-ray scattering. With both samples a volume contraction could be observed upon interaction with the tetra-D-alanine hapten. The anti-poly-D-alanyl antibodies obtained by immunization with poly-D-alanyl diphtheria toxoid showed a decrease of the volume by $10 \%$ and a decrease of the radius of gyration by $7 \cdot 7 \%$ when $90 \%$ of the binding sites were occupied by hapten. With anti-poly-D-alanyl HSA antibodies, a smaller decrease of the volume, of $3.2 \%$, and of the radius of gyration of $1.4 \%$ were found, when $62 \%$ of the binding sites were occupied. Since the other data determined for the antibodies, such as molecular weight, radii of gyration of the cross section, and form of the scattering curve, were unchanged upon interaction with hapten within the errors of measurement, it must be assumed that the change in conformation upon interaction consists in a volume contraction without significant changes in the shape. The shape of all antibodies (free and occupied by hapten) is best described by a T-shaped model. The data found for the two antibody fractions, in the free and bound states, respectively, are the following: radius of gyration of the whole particle, 6.08 to $6.00 \mathrm{~nm}$ $(-1.4 \%)$ and 6.50 to $6.00 \mathrm{~nm}(-7.7 \%)$; volume, 371 to $359 \mathrm{~nm}^{3}(-3.2 \%)$ and 399 to $358 \mathrm{~nm}^{3}(-10 \%)$.
\end{abstract}

J. Appl. Cryst. (1974). 7, 187

\section{Small-Angle Scattering Studies for Investigation of the Structure of Solid Polymers}

\author{
By E. W. Fischer ANd G. R. Strobl \\ Institut für Physikalische Chemie der Universität Mainz, Germany (BRD)
}

The recent developments of the application of small-angle scattering to structure studies of crystalline
and amorphous polymers are discussed. For the case of partially crystalline polymers a new method
for evaluating SAXS curves of samples with lamellar structure has been developed and was applied to
prove the validity of the 'two-phase' concept and to measure some properties of the so-called 'fold
surface' of polymer crystals. Taking into account the temperature dependence of SAXS, glass transition
phenomena in the disordered regions as well as premelting processes can be studied. The same method
is also used for evaluating disorder phenomena in the lamellar interfaces of paraffin crystals. Further
applications of SAXS to crystalline polymers include the study of crystallization under strain and the
dependence of SAXS on an applied stress. Amorphous polymers in the glassy or molten state
also show rather intense X-ray scattering at small angles. The origin of this scattering was investigated
in the light of recently proposed models of the structure of the amorphous phase. A specially powerful
method for solving this problem is the neutron small-angle scattering technique which is briefly reviewed. 